\title{
Predicting the probability of persistence of HIV infection with the standard model
}

\author{
Henry C. Tuckwell ${ }^{1 \dagger}$, Patrick D. Shipman ${ }^{2}$ \\ ${ }^{1}$ Max Planck Institute for Mathematics in the Sciences \\ Inselstr. 22, 04103 Leipzig, Germany \\ 2 Department of Mathematics, University of Colorado \\ Fort Collins, CO 80523-1874, USA \\ † Corresponding author: tuckwell@mis.mpg.de
}

May 21, 2018 


\begin{abstract}
We consider the standard three-component differential equation model for the growth of an HIV virion population in an infected host in the absence of drug therapy. The dynamical properties of the model are determined by the set of values of six parameters which vary across host populations. There may be one or two critical points whose natures play a key role in determining the outcome of infection and in particular whether the HIV population will persist or become extinct. There are two cases which may arise. In the first case, there is only one critical point $P_{1}$ at biological values and this is an asymptotically stable node. The system ends up with zero virions and so the host becomes HIV-free. In the second case, there are two critical points $P_{1}$ and $P_{2}$ at biological values. Here $P_{1}$ is an unstable saddle point and $P_{2}$ is an asymptotically stable spiral point with a non-zero virion level. In this case the HIV population persists unless parameters change. We let the parameter values take random values from distributions based on empirical data, but suitably truncated, and determine the probabilities of occurrence of the various combinations of critical points. From these simulations the probability that an HIV infection will persist, across a population, is estimated. It is found that with conservatively estimated distributions of parameters, within the framework of the standard 3-component model, the chances that a within host HIV population will become extinct is between $0.6 \%$ and $6.9 \%$. With less conservative parameter estimates, the probability is estimated to be as high as $24 \%$. The many factors related to the transmission and possible spontaneous elimination of the virus are discussed.
\end{abstract}

Short Title: HIV persistence in a new host

Keywords and Phrases: HIV infection, Model, Persistence

\title{
1 Introduction and theory
}

A comprehensive understanding and analysis of the early stages of HIV infection, including the process of transmission, is important as it may lead to efficient methods of reducing the probability that the virus successfully establishes itself in a new host. In a previous article (Tuckwell et al., 2008) we have addressed the problem of estimating the probability of a successful transmission of HIV infection to a new host. 
There have been several mathematical models for the growth of withinhost HIV populations, many of which are deterministic, for example Perelson et al. (1993), Phillips (1996) and Perelson et al. (1996), whereas others have incorporated chance mechanisms (Tan and Wu, 1998; Tuckwell and LeCorfec, 1998; Pearson et al., 2010). Such models have provided valuable insights into the time-course of viral dynamics and the effects of drug therapy. The simple 3-component differential equation model we examine in this article has been successfully employed to predict the temporal evolution of HIV populations in the primary stages of infection. As pointed out by Stafford et al. (2000), although some models have been hypothesized which describe the progression to acquired immunodeficiency syndrome (AIDS) (for example, Essunger and Perelson, 1994) useful information and possible prognoses can be obtained from a knowledge of the viral dynamics in the early stages. In untreated HIV infection, the risk of AIDS is known to be small until the CD4 cell count has reached low levels or the viral load has reached high levels (Phillips et al., 2001). Indeed, our approach is to examine possible outcomes predicted by a model of primary infection for various parameter values to ascertain whether the virus will be likely to persist after the initial infection period or possibly spontaneously die out due simply to dynamical system properties. It is known from clinical studies that if HIV persists into and beyond the primary stage then the baseline CD4 cell count is a major predictor of eventual outcome under highly active antiretroviral therapy (HAART) (Egger et al., 2002). Our analysis is only applicable in the absence of HAART which can decrease the plasma viral load below the limit of detection (Gallant et al., 2006). Extensions to include the effects of such therapy will be considered elsewhere.

The matter of extinction of an HIV population in an individual new host also has been raised by some authors using stochastic differential equation or similar models (Kamina et al., 2001; Merrill, 2005; Pearson et al., 2010). In this article, however, we are concerned with estimating the probability that an HIV population does in fact become established in a host after a successful transmission on the assumption that the viral population evolves deterministically according to a well-known dynamical model. The probability of persistence is determined by the distributions of the parameters which describe the host's immune response. Thus, as in accordance with the standard deterministic model, extinction does not depend on the number of virus particles which are become initially established in the new host. The veracity of such statements is contingent on the accuracy of the mathematical model, 
which due to its simplicity is only expected to give an approximate prediction of outcomes.

\section{Model description}

Dynamical modeling of the growth of HIV populations within infected hosts is complicated by spatial inhomogeneities, due for example to the occurrence of various reservoirs, such as those particularly rapidly established in lymphatic tissues (Finzi and Siliciano, 1998; Pierson et al., 2000; Pope and Haase, 2003; Kim and Perelson, 2006). However, and perhaps surprisingly, it seems that the growth of HIV populations can be satisfactorily described even when such inhomogeneities are ignored, which is the usual approach (Perelson, 2002) and the one adopted here. Some models had additional components representing resting and latently infected cells (Perelson et al, 1993; Phillips, 1996) but a relatively successful (Stafford et al., 2000) now-accepted simple time-dependent three-component model for the evolution of within-host HIV virion numbers in human or simian hosts, without any spatial variables, has been employed for the last fifteen years or so. This model (Nowak and Bangham, 1996; Bonhoeffer et al., 1997) has as component variables, at time $t, T(t)$, the number of target or activated CD $4^{+}$T-cells, $T^{*}(t)$, the number of productively infected such cells and $V(t)$ the number of free virus. In the early stages of HIV infection, to about 100 days, and in the absence of drug therapy, these quantities satisfy approximately the following three deterministic ordinary differential equations

$$
\begin{gathered}
\frac{d T}{d t}=\lambda-\mu T-k T V \\
\frac{d T^{*}}{d t}=k T V-\delta T^{*} \\
\frac{d V}{d t}=p T^{*}-c V .
\end{gathered}
$$

In Table 1 are shown the variables and parameters with their units.

In a recent article on simian immunodeficiency infection (Vaidya et al, $2010)$, an infection rate $k=k(t)$ which decreases exponentially in time to an asymptotic value has been found to give a better fit for the growth of the viral population. This aspect can be easily incorporated in the approach of this study by varying, for example, the mean of the distribution of the 
infection rate. We assume a single infection incident, alhough there may be theoretical ramifications of multiple such events (Pujol et al, 2009).

Table 1: Variables and parameters

\begin{tabular}{|c|c|c|}
\hline Symbol & Description & Units \\
\hline $\mathrm{T}$ & Density of target CD $4+\mathrm{T}$ cells & T-cells $\mu \mathrm{l}^{-1}$ \\
\hline $\mathrm{T}^{*}$ & Density of productively infected CD4+ T cells & $\mathrm{T}^{*}$-cells $\mu \mathrm{l}^{-1}$ \\
\hline $\mathrm{V}$ & Density of virions & Virions $\mu \mathrm{l}^{-1}$ \\
\hline$\lambda$ & Rate of arrival of target $\mathrm{CD} 4+\mathrm{T}$ cells & T-cells $\mu \mathrm{l}^{-1}$ day $^{-1}$ \\
\hline$\mu$ & Per capita rate of decrease of target $\mathrm{CD} 4+\mathrm{T}$ cells & day $^{-1}$ \\
\hline$k$ & Rate of conversion of $\mathrm{T}$ to $\mathrm{T}^{*}$ by virus & $\operatorname{day}^{-1}\left(\text { virions } \mu \mathrm{l}^{-1}\right)^{-1}$ \\
\hline$\delta$ & Per capita rate of decrease of productively infected $\mathrm{CD} 4+\mathrm{T}$ cells & day $^{-1}$ \\
\hline$p$ & Rate at which $\mathrm{T}^{*}$ cells produce virus & virions $\mathrm{T}^{*}$ cells ${ }^{-1}$ day $^{-1}$ \\
\hline$c$ & Per capita rate of decrease of virions & day $^{-1}$ \\
\hline
\end{tabular}

\subsection{Equilibrium analysis}

Equilibrium point analysis of the system of differential equations (1)-(3) has been carried out by several authors (for example Bonhoeffer et al. (1997), Stafford et al., 2000, Tuckwell and Wan, 2000). There are two equilibrium points, denoted by $P_{1}$ and $P_{2}$. These occur at

$$
P_{1}=\left(\frac{\lambda}{\mu}, 0,0\right)
$$

and

$$
P_{2}=\left(\frac{c \delta}{k p}, \frac{\lambda}{\delta}-\frac{c \mu}{k p}, \frac{\lambda p}{c \delta}-\frac{\mu}{k}\right) .
$$

To discuss the outcomes for an infection by HIV, define

$$
R=\frac{c \delta \mu}{k \lambda p}
$$

and note the following possibilities. 


\subsubsection{Case 1, $R>1$.}

It is clear that in this case the equilibrium values of $T^{*}$ and $V$ are negative so that $P_{2}$ is outside the first octant at unbiological values. This might occur, for example, if the if the arrival rate of target CD $4+\mathrm{T}$ cells from the thymus is sufficiently small to make

$$
\lambda<\frac{c \mu \delta}{k p} .
$$

Alternatively, large enough values of one or more of $c, \mu$ and $\delta$ and/or small enough values of one or both of $k$ and $p$, will also tend to make this inequality hold. Effectively then there is just one critical point $P_{1}$ which is at zero virions $(V=0)$ and zero productively infected cells $\left(T^{*}=0\right)$ with the unperturbed equilibrium value of target CD4+ $\mathrm{T}$ cells $T=\frac{\lambda}{\mu}$. Thus

$$
R>1 \Longrightarrow \lim _{t \rightarrow \infty} V(t) \rightarrow 0 .
$$

This means that, according to the model, the virus goes extinct and the infected host is cleared of the HIV virus. If the host immune system parameters do not change as a consequence of the infection, then a second dose of virions would meet also with extinction and this process could, theoretically, be repeated indefinitely.

\subsubsection{Case 2, $R<1$}

In this case both critical points $P_{1}$ and $P_{2}$ are at biologically meaningful values in the first octant. The point $P_{1}$ is an unstable saddle point and the point $P_{2}$ is an asymptotically stable spiral point. Thus when case 2 conditions are fulfilled,

$$
\lim _{t \rightarrow \infty} V(t)=V_{f}>0
$$

where

$$
V_{f}=\frac{\lambda p}{c \delta}-\frac{\mu}{k}
$$

is the equilibrium value of the number density of virions.

Note that in Tuckwell and Wan (2000) the possibility of an extra term $-k T V$ in (3) was considered leading to the replacement of $p$ by $p-\delta$ in the expression for $R$. However, since $\delta<<p$, the additional term, which was taken into account in the calculations presented below, makes very little difference in determining the probabilities which we shall calculate. Note 
that at $R=0$, the bifurcation point, there is only one critical point $P_{1}$ so that $V \rightarrow 0$ as in Case 1 (Tuckwell and Wan, 2000).

It is apparent that factors which make $R$ smaller, promote the persistence of the diseased state and factors which make $R$ larger inhibit the host viral population. From the definition of $R$ it is clear that larger values of the following promote the persistence of HIV infection: $k$, leading to greater frequency of virus-T-cell interactions; $\lambda$, giving a larger density of target T-cells; and $p$, the number of virions produced per $\mathrm{T}^{*}$-cell. Similarly, larger values of the following tend to inhibit the HIV population: $c$, the viral clearance rate; $\delta$, the rate of disappearance of $\mathrm{T}^{*}$-cells; and $\mu$, the rate of disappearance of target T-cells. We use the term promoters for $k, \lambda$ and $p$ and we call $c, \delta$ and $\mu$, inhibitors, these two groups being treated differently in the analysis below.

\section{Methods}

Let us denote the random variables representing the parameters by the symbols given in Table 2 .

Table 2: Notation for random parameters

\begin{tabular}{|c|c|}
\hline Parameter & Random \\
\hline$\lambda$ & $\Lambda$ \\
$\mu$ & $\mathrm{M}$ \\
$k$ & $K$ \\
$p$ & $\mathrm{P}$ \\
$\delta$ & $\Delta$ \\
\hline
\end{tabular}

Whether case 1 or case 2 applies depends on the values of the set of 6 random variables

$$
U=\{\Lambda, M, K, \Delta, P, C\} .
$$

The calculation which we address in this article is the determination of the probability of occurrence of the various values of $U$. In particular we will attempt to estimate

$$
p_{E}=\operatorname{Prob}\{U \in E\}
$$


where $E$ is the set of values of the 6 parameters which lead to $P_{1}$ being an asymptotically stable node. This will provide an estimate of the probability that the virus goes extinct, even in the absence of any drug treatment. Note that this is a population probability describing the chance that the virus does not persist in a randomly selected member of the population of hosts, not a probability that in a given individual the virus will go extinct. That is, if the host population size is $n$ and $N_{R}$ is the number who recover from the viral infection without treatment then

$$
\mathrm{E}\left(N_{R}\right)=N p_{E}
$$

\subsection{Estimation of $p_{E}$}

All the parameters in $U$ are non-negative and continuous so that they may be ascribed probability density functions. Let us denote the densities of the six components by

$$
f_{\Lambda}, f_{M}, f_{K}, f_{\Delta}, f_{P}, f_{C}
$$

It is feasible to determine $p_{E}$ analytically if certain simplifying assumptions are made about the distributions of the various parameters. However, a better approach is to be guided by empirical evidence to estimate the various densities $f$ and then use simulation to find $p_{E}$.

Stafford, et. al. (2000) gave estimates (their Table 2) for the values of the parameters $\mu, k, \delta$ and $p$ for 10 patients infected by HIV. In Table 3, we give the means, standard deviations, and upper and lower $95 \%$ confidence limits for the means as well as the maxima and minima for these four parameters. As in Stafford et al. it is assumed that $c=3$ for all patients and also that $\lambda=10 \mu$. The latter is consistent with Stafford et al.'s values for $R_{0}$ (which is the reciprocal of $R$ defined in (6)). However, different values of $\lambda$ are obtained using Stafford et al.'s equilibrium value for $T=T_{s s}$. Thus using $\lambda=10 \mu$, the mean is $\bar{\lambda}=0.1089$ whereas using the values of $T_{s s}$ one obtains $\bar{\lambda}=0.193$. In the simulations below we use the values $\lambda=10 \mu$.

We note that based on the Stafford et al. (2000) data, a 95\% confidence interval for the mean of $R$ is $(0.0710,0.1409)$ and so the chances of $V_{f}=0$ is practically zero, as would be expected from a group of patients who definitely have a sustained HIV infection. The same conclusion arises from the $95 \%$ confidence interval $(4.089,7.310)$ for the mean value of $R_{0}$. 
Table 3: Some statistics for 10 patients, from Stafford et al. (2000)

\begin{tabular}{|c|c|c|c|c|}
\hline & $\mu$ & $k$ & $\delta$ & $p$ \\
\hline Mean & 0.01089 & $1.179 \times 10^{-3}$ & 0.3660 & 1426.8 \\
\hline Standard deviation & 0.005727 & $1.422 \times 10^{-3}$ & 0.193 & 2049.36 \\
\hline Minimum & 0.0043 & $0.19 \times 10^{-3}$ & 0.13 & 98 \\
\hline Maximum & 0.020 & $4.80 \times 10^{-3}$ & 0.80 & 7100 \\
\hline Upper 95\% conf limit & 0.00734 & $0.2976 \times 10^{-3}$ & 0.246377 & 156 \\
\hline Lower 95\% conf limit & 0.0144396 & $2.560 \times 10^{-3}$ & 0.4856 & 2697 \\
\hline
\end{tabular}

\subsection{Distributions of parameters}

For the estimation of the probability that the virus goes extinct after infecting a new host, the distributions of the random variables $\Lambda, M, K, \Delta, P, C$ are required. These are not known with certainty so we assume that, by a centrallimit theorem argument, the parameters wil be approximately normally distributed, although lognormal distributions were previously employed (Ciupe et al., 2006). For the density of $C$ we take a delta-function concentrated at $c=3$. If we take the remaining variables $\Lambda, M, K, \Delta, P$ to have normal distributions then there is always going to be a small probability mass for each variable at values less than zero, which is biologicaly unrealistic for the present set of parameters. Hence we have chosen truncated normal distributions. For a normal random variable $X$ with mean $m$, standard deviation $\sigma$, truncated to be on the interval $(\alpha, \beta)$, the probability density function is (Johnson and Kotz, 1970)

$$
f_{X}(x)=\frac{\phi\left(\frac{x-m}{\sigma}\right)}{\sigma\left[\Phi\left(\frac{\beta-m}{\sigma}\right)-\Phi\left(\frac{\alpha-m}{\sigma}\right)\right]}, \alpha<x<\beta,
$$

where $\phi$ and $\Phi$ are the density and distribution function for a standard normal random variable. The mean of the truncated variable is

$$
E[X]=m+\frac{\phi\left(\frac{\alpha-m}{\sigma}\right)-\phi\left(\frac{\beta-m}{\sigma}\right)}{\left[\Phi\left(\frac{\beta-m}{\sigma}\right)-\Phi\left(\frac{\alpha-m}{\sigma}\right)\right]} \sigma
$$

which is useful for comparing with the mean of the parent distribution. 


\subsection{Sampling procedures}

In order to estimate $p_{E}$ we generate samples of size 10,000 for each of the parameters $\mu, \lambda, k, \delta, p$ using the truncated normal probability density functions. The distributions are specifed in terms of the four quantites: mean, standard deviation (both chosen before truncation), and the lower and upper truncation points. For the upper and lower bounds, three approaches were used.

Method 1: Use the means and standard deviations of the Stafford et al. (2000) data of Table 3 and use the minimum value of each parameter as $\alpha$ and the maximum value as $\beta$ for the corresponding random variable.

Method 2: Take $\alpha$ to be half of the minimum value of the Stafford et al. (2000) data, and $\beta$ as twice the maximum value of the Stafford et al. (2000) data. The motivation here is to extend the ranges of the parameter data of Stafford et al. (2000), because those data are for patients who became infected and remained infected, whereas some members of the population may become infected with HIV and recover spontaneously if their immune system is able to eliminate the virus as in the case of $R>1$. Again we use the original standard deviation given in Table 3. However, instead of the actual mean, in order to examine outcomes with more extreme but possible parameter values, we may also use the estimated lower and upper confidence limits of the means.

Method 3: Here the means of the parameters which are promoters, $k, \lambda, p$ are multiplied by 0.9 , whereas the means of the parameters $\delta$ and $\mu$ which are inhibitors are multiplied by 1.1. The same standard deviations as in Method 1 are employed and the $95 \%$ confidence limits for the means are recalculated. We do this using the same sets of lower and upper bounds for the parameter densities as for Method 2.

Method 4: This method is also motivated by the shifting of the parameter distributions of the Stafford et al. (2000) to account for bias in the data due to the fact that they were taken from patients in whom HIV persisted. Here we multiply the means and the lower and upper bounds (taken to be the minimum and maximum values of the parameters in Table 3, as in Method 1 ) of the promoter parameters $k, \lambda, p$ by values $s_{p}<1$, and we multiply the means and the upper and lower bounds of the inhibitor parameters $\delta$ and $\mu$ by values $s_{d}>1$. Such choices must lead to a greater chance of extinction

of the virus. In all cases the value of $R$, which is now a random variable, is computed, and hence the probability $p_{E}$ that $P_{1}$ is an asymptotically stable 
node or equivalently that extinction of the host HIV population occurs, can be estimated.

\section{Results}

For Method 1, the use of the means and standard deviations of the data in Table 3 as well as the minima and maxima as the truncation limits resulted in the following means from formula (16) for the parameters: (with original means in brackets $) E[M]=0.0114(0: 01089), E[K]=1.6401 \times 10^{-3},(1.179 \times$ $\left.10^{-3}\right), E[\Delta]=0.3965(0.3660)$ and $E[P]=2141.1(1426.8)$. Thus, the truncation procedure results in increases in the means for all of these 4 parameters.

\subsection{Calculated extinction probabilities}

In total, as described above for Methods 1,2 and 3, there were many different ways in which the parameters of the distributions of the randomized parameters were chosen. To illustrate, there are shown in Figure 1, the histograms for the parameter $\lambda$ with truncated distributions according to Methods 1-3.
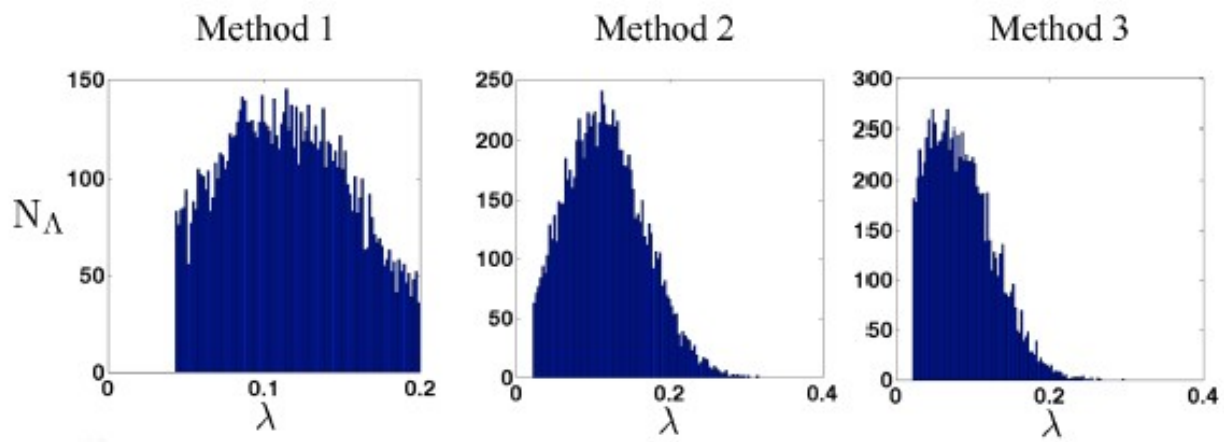

Figure 1: The truncated normal distributions of $\Lambda$ according to the densities described in the text, with means chosen to be the values of Table 3.

For each Method and for each choice of parameterization of the distributions the calculated results for the probabilities of extinction $p_{E}$ were as follows (Table 4). 
Table 4: Estimated extinction probabilities, $p_{E}$

\begin{tabular}{|c|c|c|c|}
\hline & Method 1 & Method 2 & Method 3 \\
\hline Maximum value & 0.0685 & 0.1354 & 0.1376 \\
\hline Minimum value & 0.0063 & 0.0066 & 0.0158 \\
\hline Average value & 0.0248 & 0.0450 & 0.0693 \\
\hline
\end{tabular}

Figures 2 and 3 show geometrically, by means of three-dimensional scatter diagrams, the situation with regard to the critical points in two extreme cases. If $P_{2}$ falls outside the first octant then spontaneous recovery can, according to the model predictions, occur. In Figure 2 is shown the spatial distribution of $P_{1}$ and $P_{2}$ values when the probability of recovery is small, with most $P_{2^{-}}$ values falling in the first octant. In contrast, Figure 3 shows a case where the probability of recovery is much higher as many more $P_{2}$-values fall outside of the first octant. The sample sizes used for these figures were reduced to 2000 from the usual 10000 to make the figure files manageable.

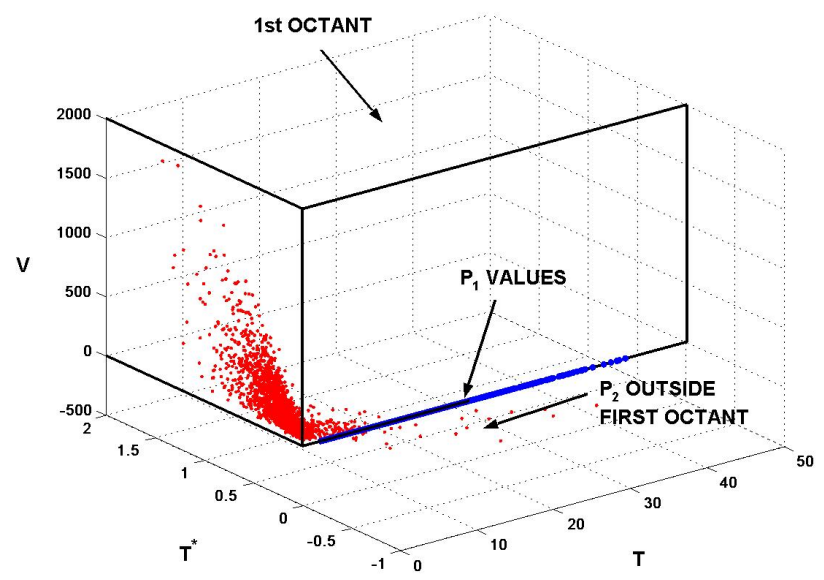

Figure 2: A sample distribution of the positions in $(T, T *, V)$-space of the two critical points $P_{1}$ (blue circles) and $P_{2}$ (red circles) when the probability of recovery from HIV infection is very small. Only a relatively small number (about $1.5 \%$ ) of points $P_{2}$ lie outside the first octant. Here the sample is generated by Method 1 using the actual minima, mean and maxima as given in Table 3 for the parameters of Stafford et al. (2000) for use in the truncated normals. Sample size 2000. 
Table 5: Values of $p_{E}$, Method 4.

\begin{tabular}{|c|c|c|}
\hline$s_{d}$ & $s_{p}$ & $p_{E}$ \\
\hline 1.1 & 0.9 & 0.9747 \\
1.2 & 0.8 & 0.9499 \\
1.25 & 0.75 & 0.9329 \\
1.3 & 0.7 & 0.9160 \\
1.4 & 0.6 & 0.8586 \\
1.5 & 0.5 & 0.7601 \\
\hline
\end{tabular}

We also explore calculations in which we shift the distributions to account for bias in the Stafford data towards patients in which the virus persisted, which we have called Method 4. Results are given in Table 5 and see also Figure 3.

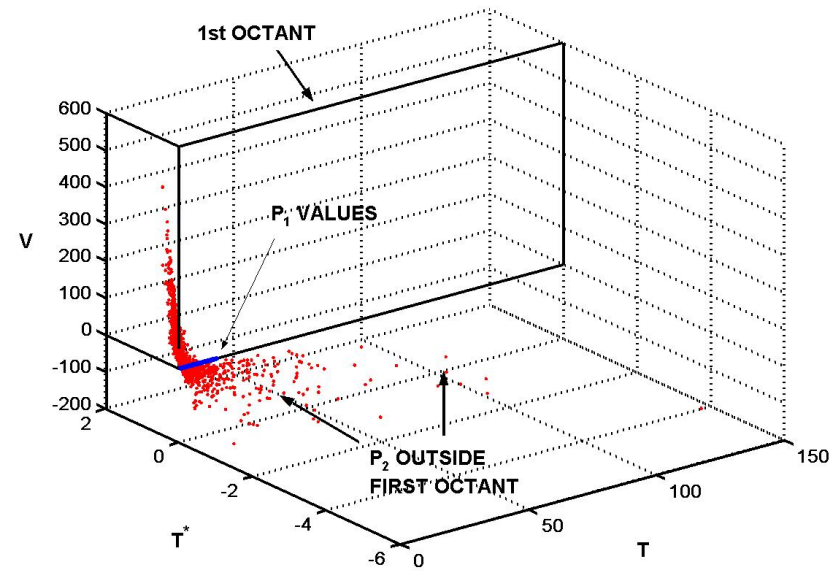

Figure 3: A sample distribution of the positions in $(T, T *, V)$-space of the two critical points $P_{1}$ (blue circles) and $P_{2}$ (red circles) when the probability of recovery from HIV infection is relatively large. About $25 \%$ of the points $P_{2}$ lie outside the first octant. Here the sample is generated using Method 4 where the means and upper and lower truncation points for the distributions for $k, \lambda, p$ are multiplied by 0.5 and those of $\mu$ and $\delta$ are multiplied by 1.5 . Sample size 2000. 


\section{Discussion}

The probabilities $p_{E}$ determined above represent the chance that the parameters of the virus-immune system dynamics are such that the only equilibrium is at zero virions and hence the virus would, theoretically, be eliminated without medical intervention. The values of $p_{E}$ given in Table 4 have approximate upper bounds of 0.07 for Method 1 and 0.14 for Methods 2 and 3. These probabilities are quite small, which is due, at least in part, to the fact that the estimated parameter distributions are biased, being derived from a sample of individuals, all of whom were infected by HIV for tracked periods of from 46 days to several hundred days. A histogram of steady state $V=V_{f}$ values for Method 1 is shown in Figure 4. Here a small fraction of values is less than or equal to zero, and ones just greater than zero could be driven to zero with noise.

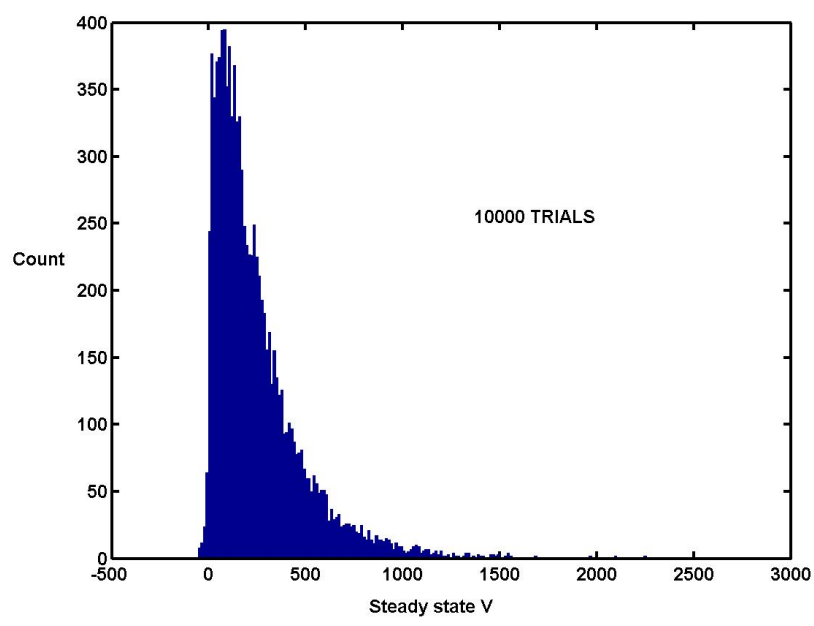

Figure 4: Histogram of steady state $V$ values when the parameter distributions are obtained by the conservative method 1 .

When the ranges of the parameters were extended by not extreme amounts in an attempt to counter the bias towards an infected group, values of $p_{E}$ were obtained as high as about 0.24. Thus it is feasible that in the general population, within the framework of the standard 3-component model, that a considerable percentage of individuals could be infected by HIV but the virus would subsequently be eliminated without intervention which would constitute spontaneous recovery. 
It is noted that the virus population in patient 3 of the Stafford et al. data had dwindled to $0.1 \%$ of its maximum after 50 days. Furthermore, if one takes the values of the parameters for all 10 patients which lead to the largest value of $R=R_{\max }$, which are $\delta=0.8, k=0.00019, p=98$ and the standard values $c=3$ and $\mu / \lambda=0.1$, then $R_{\max }=12.9$ which far exceeds the value required to make the only equilibrium point the one at zero virions.

The probability of transmission of HIV has been estimated in various groups. Gray et al. (2001) found an average value of 0.0011 per coital act in heterosexual couples in Rakai, Uganda and Wawer et al. (2005) found a value of 0.0082 for a similar population. See also Chakraborty et al. (2001). Higher values, around 0.031 , had been obtained for a group of male military trainees who interacted with female prostitutes in Thailand (Mastro et al., 1994). In the Ugandan studies, two of the main factors influencing transmission were time since infection of the index partner and degree of ulceration.

Many findings in such articles, and in Downs et al. (1996), concern transmission in stable monogamous discordant couples, in which one partner is HIV-positive and yet, despite frequent and long-term possibilities of transmission, the HIV-negative partner does not seroconvert, even in the absence of condom use. This was also reported in the metaanalytical study of Powers et al. (2008), where there was found to be no transmission even after hundreds of contacts between members of discordant couples. Similarly, transmission had been remarkably found to not occur in some individuals despite multiple high-risk sexual exposures (Rowland-Jones, 1995; Paxton et al., 1996). In the latter study, CD8+ lymphocytes were found to have greater anti-HIV-1 activity than those from nonexposed controls. There is always the possibility that the non-transmission of the virus in discordant couples is due to properties of the infected partner, such as a low viral load. Nevertheless, it is feasible, though considered unlikely, by HIV experimentalists and theorists, that in some of these long-term discordant couples, the non-index partner does become infected briefly, possibly repetitively, but the virus is subsequently eliminated as predicted by the standard model. The pe$\operatorname{riod}(\mathrm{s})$ of infection could be brief and the viral load small so that the partner remained asymptomatic.

According to Haynes et al. (1996) the existence of individuals who have been exposed multiple times to HIV and are persistently HIV-seronegative raises the possibility that a small percentage of them may be resistant to HIV, or may have been able to clear the infection. The situation is made less clear by virtue of the issues of viral loads below threshold for detection and 
other false negatives. Also pertinent are the rates of HIV infection in children born to HIV-infected parents. According to an analysis of Rowland-Jones et al. (1993), 60-85\% of children exposed either before or after birth to HIV were not infected. In a European study (Nöstlinger et al., 2004), a study of $165 \mathrm{HIV}$-affected families with 279 children, found that $68 \%$ of the children were HIV-negative.

Much has been written about host immune properties or properties of the virus which may lead to the fending off of HIV infection (Haynes et al., 1996; Lo Caputo et al., 2003) or as in the case of elite controllers (sometimes called long term non progressors or just HIV controllers) who constitute about 5$10 \%$ of cases, maintaining low viral burdens and not converting to the AIDS regime. Genetic factors such as the gene encoding CCL3L1 have been shown to affect susceptibility to AIDS (Gonzalez et al., 2005) and certain mutations in the gene encoding the protein CCR5 afford immunity to HIV in mice (Holt et al, 2010).

There are evidently, however, no documented cases of clearance of HIV from an an individual with an established infection (Alan Perelson and Marc Pellegrini, personal communications). There were cases reported in the press of changes in individuals from HIV-positive to HIV-negative, the most noteworthy being that of Alan Stimpson. The standard model employed here predicts, under reasonable assumptions on the distributions of parameters, that a small percentage of the population might be able to clear HIV after infection. As pointed out above, it would be hard to verify or refute this prediction if the duration of infection and the viral load were both small. In actuality, there are several immune system components omitted in the standard 3-component model, such as latently-infected cells, cytotoxic T-cells (CD8+ T-cells, CTL), natural killer cells and dendritic cells. The incorporation of these additional components would make the mathematical modeling much more complicated but may yield more insight into the possibility of an individual's clearing an HIV infection.

\section{Acknowledgements}

We thank Dr Alan Perelson of Theoretical Biology \& Biophysics, Los Alamos National Laboratory, Los Alamos, New Mexico, USA, and Dr Marc Pellegrini of the Walter and Eliza Hall Institute of Medical Research, Parkville, Victoria, Australia, for useful correspondence. 


\section{References}

Bonhoeffer N Coffin JM and Nowak MA (1997). Human Immunodefciency virus drug therapy and virus load. J Virology 71, 3275-3278.

Chakraborty $\mathrm{H}$ et al. (2001) Viral burden in genital secretions determines male-to-female sexual transmission of HIV-1: a probabilistic empiric model. AIDS 15: 621-627.

Ciupe MS et al. (2006) Estimating kinetic parameters from HIV primary infection data through the eyes of three different mathematical models. Math Biosci 200: 1-27.

Downs AM et al. (1996) Probability of heterosexual transmission of HIV: relationship to the number of unprotected sexual contacts. JAIDS 11: 388-395.

Egger M et al. (2002) Prognosis of HIV-1-infected patients starting highly active antiretroviral therapy: a collaborative analysis of prospective studies. The Lancet 360, 119-129.

Essunger, P and Perelson, A.S. (1994). Modeling HIV infection of CD4+ T-cell subpopulations. J. Theor. Biol. 170: 367-391

Finzi D, Siliciano RF (1998) Viral Dynamics in HIV-1 Infection. Cell 93, 665-671.

Gallant JE et al. (2006) Tenofovir DF, emtricitabine, and efavirenz vs. zidovudine, lamivudine, and efavirenz for HIV. NEJM 354, 251-260.

Gonzalez E et al. (2005) The influence of CCL3L1 gene-containing segmental duplications on HIV-1/AIDS susceptibility. Science 307: 1434-1440.

Gray RH et al. (2001) Probability of HIV-1 transmission per coital act in monogamous, heterosexual, HIV-1-discordant couples in Rakai, Uganda. Lancet 357: 1149-1153.

Holt $\mathrm{N}$ et al. (2010) Human hematopoietic stem/progenitor cells modified by zinc-finger nucleases targeted to CCR5 control HIV-1 in vivo. Nat Biotech 28: 839-847.

Johnson N, Kotz S (1970) Distributions in Statistics: Continuous Univariate Distributions 1. Houghton Mifflin, New York.

Kamina A, Makuch RW, Zhao H (2001) A stochastic modeling of early HIV-1 population dynamics. Math Biosci 170: 187-198.

Kim H, Perelson AS (2006) Dynamic characteristics of HIV-1 reservoirs. Curr Opin HIV AIDS 1, 152-156. 
Lo Caputo S et al. (2003) Mucosal and systemic HIV-1-specific immunity in HIV-1-exposed but uninfected heterosexual men. AIDS 17: 531-539.

Mastro TD et al. (1994) Probability of female-to-male transmission of HIV-1 in Thailand. Lancet 343: 204-207.

Merrill SJ (2005) The stochastic dance of early HIV infection. J. Comp Appl Math 272: 74-79.

Nöstlinger C et al. (2004) Families affected by HIV: parents' and children's characteristics and disclosure to the children. AIDS Care 16: 641-648.

Nowak MA, Bangham CRM (1996). Population dynamics of immune responses to persistent viruses. Science 272, 74-79.

Paxton WA et al. (1996) Relative resistance to HIV1 infection of CD4 lymphocytes from persons who remain uninfected despite multiple high-risk sexual exposures. Nat Med 2: 412-417.

Pearson JE et al. (2010) Stochastic theory of early viral infection: continuous versus burst production of virions. PLoS Comp Biol Volume 7: e1001058.

Perelson AS (2002) Modelling viral and immune system dynamics. Nat Rev Immunol 2, 28-36.

Perelson AS, Kirschner DE, De Boer R (1993) Dynamics of HIV infection of CD4 T cells. Math Biosci 114: 81-125.

Perelson AS, Neumann AU, Markowitz M et al. (1996) HIV-1 dynamics in vivo: virion clearance rate, infected cell life-span, and viral generation time. Science 271: 1582-1586.

Phillips AN (1996) Reduction of HIV concentration during acute infection: idependence from a specific immune response. Science 271: 497-499.

Phillips AN et al. (2001) HIV viral load response to antiretroviral therapy according to the baseline CD4 cell count and viral load. JAMA 286: 2560-2567.

Pierson T, McArthur J, Siliciano RF (2000) Reservoirs for HIV-1: mechanisms for viral persistence in the presence of antiviral immune responses and antiretroviral therapy. Ann Rev Immunol 18: 665-708.

Pope M, Haase AT (2003) Transmission, acute HIV-1 infection and the quest for strategies to prevent infection. Nat Med 9: 847-852.

Powers KA et al. (2008) Rethinking the heterosexual infectivity of HIV-1: a systematic review and meta-analysis. Lancet Infect Dis 8: 553-563.

Pujol JM et al. (2009) The effect of ongoing exposure dynamics in dose response relationships. PLoS Comp Biol 5: e1000399. 
Rowland-Jones S et al. (1993) HIV-specific cytotoxic T-cell activity in an HIV-exposed but uninfected infant. Lancet 341: 860-861.

Rowland-Jones S et al. (1995) HIV-specific cytotoxic T-cells in HIV-exposed but uninfected Gambian women. Nat Med 1: 59-64.

Stafford MA et al. (2000) Modeling plasma virus concentration during primary HIV infection. J Theor Biol 203: 285-301.

Tan WY, Wu H (1998) Stochastic modeling of the dynamics of CD4+ Tcells infection by HIV and some Monte-Carlo studies. Math Biosci 147: 173-205.

Tuckwell HC, Le Corfec E (1998) A stochastic model for early HIV-1 population dynamics. J Theor Biol 195: 451-463.

Tuckwell HC, Shipman PD, Perelson AS (2008) The probability of HIV infection in a new host and its reduction with microbicides. Math Biosci 214: 81-86.

Tuckwell HC, Wan FYM (2000) Nature of equilibria and effects of drug treatments in some simple viral population dynamical models. IMA J Math Appl Med Biol 17: 311-327.

Vaidya RM et al. (2010) Viral dynamics during primary simian immunodeficiency virus infection: effect of time-dependent virus infectivity. J Virol 84: 4302-4310.

Wawer MJ et al. (2005) Rates of HIV-1 transmission per coital act, by stage of HIV-1 infection, in Rakai, Uganda. J Infect Dis 191: 1403-1409. 